\title{
Flora arbórea do Cerrado de Mato Grosso do Sul
}

\author{
Marcelo Leandro Bueno', Ary Teixeira de Oliveira-Filho², \\ Vanessa Pontara ${ }^{1}$, Arnildo Pott ${ }^{3} \&$ Geraldo Alves Damasceno-Junior ${ }^{3}$
}

\author{
'Universidade Federal de Viçosa, Laboratório de Ecologia e Evolução de Plantas, Departamento de Botânica, \\ Av. PH Rolfs, CEP 36570-000, Viçosa, MG, Brasil. buenotanica@gmail.com \\ 2Universidade Federal de Minas Gerais, Programa de Pós-graduação em Biologia Vegetal, Departamento de Botânica, \\ Av. Antônio Carlos, 6627, CEP 31270-901, Belo Horizonte, MG, Brasil. \\ 3Universidade Federal do Mato Grosso do Sul, Laboratório de Botânica, Instituto de Biociências, \\ Cidade Universitária s/n, CEP 79070-900, Campo Grande, MS, Brasil.
}

RESUMO - A principal unidade de vegetação do Mato Grosso do Sul é o Cerrado. Por apresentar um acelerado processo de perda de habitat associado à baixa taxa de conhecimento da flora do estado, torna-se de suma importância o conhecimento das espécies arbóreas registradas neste Bioma. Neste trabalho apresentamos uma lista da composição das espécies arbóreas do Cerrado no Mato Grosso do Sul, com o objetivo de subsidiar futuras avaliações sobre o estado de conservação e implementação de propostas que auxiliem na conservação dos seus recursos naturais. Os dados foram extraídos do NeoTropTree, que consiste em um conjunto de banco de dados de listas florística de espécies arbóreas. Foram registrados 546 espécies arbóreas distribuídas em 84 famílias botânicas e 255 gêneros, sendo que três espécies são exclusivas do estado.

Palavras-chave: Cerrado sul mato-grossense, diversidade, espécies arbóreas, Savana Neotropical

\begin{abstract}
Tree flora of the Cerrado of Mato Grosso do Sul state. The main unit of Mato Grosso do Sul vegetation is the Cerrado. For presenting an accelerated habitat loss process associated by low rate of flora knowledge, becomes of paramount importance the knowledge on tree species in this Biome. In this work, we to present a list of tree species of the Cerrado in Mato Grosso do Sul, in order to support future assessments of the state of conservation and implementation of proposals that can help to conserve its natural resources. The data were extracted from NeoTropTree, which consists of a database set of floristic lists of tree species. We recorded 541 tree species in 85 plant families and 252 genera, and three species are exclusive to the state.
\end{abstract}

Keywords: Cerrado south mato-grossense, diversity, Neotropical Savanna, tree species

\section{INTRODUÇÃO}

O Cerrado é uma savana neotropical e o segundo maior bioma da América do Sul, ocupando uma área cerca de 2 milhões $\mathrm{km}^{2}$, ou $22 \%$ do território nacional. A sua área contínua incide sobre os estados de Goiás, Tocantins, Mato Grosso, Mato Grosso do Sul, Minas Gerais, Bahia, Maranhão, Piauí, Rondônia, Paraná, São Paulo e Distrito Federal, além dos encraves no Amapá, Roraima e Amazonas (Ribeiro \& Walter 2008). Além disso, incorpora ainda partes do território de países vizinhos como Bolívia e Paraguai (Ribeiro \& Walter 2008). Apesar de sua aparente uniformidade, o Cerrado abriga nas diversas fitofisionomias uma grande variedade de espécies, sendo reconhecido como a savana mais rica do mundo, com uma flora com mais de 11.000 espécies de plantas nativas (Mendonça et al. 2008), das quais 4.400 são endêmicas (Myers et al. 2000), e estima-se que $1 / 3$ da biota brasileira e cerca de $5 \%$ da fauna e flora mundial (Ribeiro \& Walter 2008).

O Cerrado é o bioma brasileiro que mais sofreu alterações com a ocupação humana, posteriormente à Mata Atlântica, decorrente da crescente pressão para a abertura de novas áreas, visando incrementar a produção de carne e grãos para exportação, acarretando um progressivo esgotamento dos recursos naturais do bioma Cerrado (Fundo Mundial para a Natureza 1995, Ministério do Meio Ambiente 2009). Resultados apresentados pelo Ministério do Meio Ambiente em 2009, indicavam a existência de uma área de cobertura vegetal nativa da ordem de 1.043.346,02 $\mathrm{km}^{2}$, o que equivale a $51,16 \%$ da área natural do bioma. $\mathrm{O}$ avanço indiscriminado do desmatamento, sem um plano de manejo sustentado tem ameaçado substancialmente a elevada biodiversidade do Cerrado, onde penas $11 \%$ da área de cobertura do bioma Cerrado se encontram em áreas protegidas (Scarano et al. 2014). Tais fatores atribuíram ao Cerrado como um dos hotspots mundiais de biodiversidade (Mittermeier et al. 1999, Myers et al. 2000, Silva \& Bates 2002, Ministério do Meio Ambiente 2009).

No estado de Mato Grosso do Sul, o Cerrado ocupava mais de $60 \%$ do território restando menos de $25 \%$ (Ministério do Meio Ambiente 2009), além de exibir o título de um dos estados brasileiros com menores taxas de coleções botânicas por quilômetro quadrado (Peixoto 2003, Shepherd 2003, Alves et al. 2017), apresentando extensas áreas com baixas ou insignificantes índices de coleta (Martinelli \& Martins 2010, Alves et al. 2017). A vegetação de Mato Grosso do Sul, além da área predominante de Cerrado, tem relações fitogeográficas com Chaco, Amazônia, Mata Atlântica, o 
que caracteriza uma diversificação na riqueza de espécies (Rizzini 1979). Sendo que o Chaco penetra pelo sudoeste e a Amazônia pelo noroeste, ambos principalmente no Pantanal, enquanto espécies da Mata Atlântica vem do sul e do leste (Pott \& Pott 2003).

Ao considerar que a principal unidade de vegetação do Mato Grosso do Sul é o Cerrado, e que o estado apresenta um processo de perda de habitat, associado a baixa taxa de conhecimento da flora do estado, torna-se de suma importância o conhecimento das espécies arbóreas registradas neste Bioma. Deste modo, o objetivo deste trabalho é apresentar uma listagem de espécies arbóreas registradas no Cerrado do estado de Mato Grosso do Sul para que sirva como subsídio nas futuras avaliações sobre o estado de conservação deste Bioma, bem como para a implementação de propostas que auxiliem na conservação dos seus recursos naturais.

\section{MATERIAL E MÉTODOS}

O bioma Cerrado apresenta duas estações distintas em relação a distribuição anual das chuvas: seca e chuvosa, sendo que a precipitação média anual de mais de $90 \%$ da área situa-se na faixa de 800-2000 mm (Ab'Saber 2003). Este bioma apresenta, em geral, solos distróficos ácidos e com altos níveis de alumínio (Furely \& Ratter
1988, Haridasan 2000). É dominado por Latossolos (Lopes 1984, Adámoli et al. 1987, Reatto et al. 1998), mas um número de diferentes tipos de solos e uma ampla gama de características químicas e físicas podem ocorrer. O estado de Mato Grosso do Sul (MS) localizado no centrooeste do Brasil, é representado por três diferentes biomas: Cerrado (savana) como o bioma dominante, Pantanal (incluindo parte do Chaco) e parte da Mata Atlântica lato sensu (Instituto Brasileiro de Geografia e Estatística-IBGE 2004). O estado do MS apresenta uma média de altitude variando entre 200 a 600 metros, e o clima predominante é o tropical, com duas estações bem definidas, uma chuvosa (verão) e outra seca (inverno), com índices pluviométricos chegam a $1.500 \mathrm{~mm}$ ao ano. Nesses locais a temperatura média varia de acordo com o relevo, pois nas partes mais baixas a temperatura média anual é de $26^{\circ} \mathrm{C}$, enquanto que nos planaltos é de $23^{\circ} \mathrm{C}$.

A lista da flora arbórea para o bioma Cerrado do MS está representada em 39 áreas (Fig. 1) e 2845 espécimes extraídas do NeoTropTree (Oliveira-Filho 2014). As áreas determinadas para compor esta lista ocorrentes no bioma Cerrado no MS representam as seguintes fisionomias de acordo com Oliveira-Filho (2009): cerradão mesotrófico 18 áreas, cerrado stricto sensu (16), cerradão distrófico (4) e cerrado rupícola (1) (Fig. 1). Foram também inserido na lista, ocorrência de espécies em razão do conhecimento

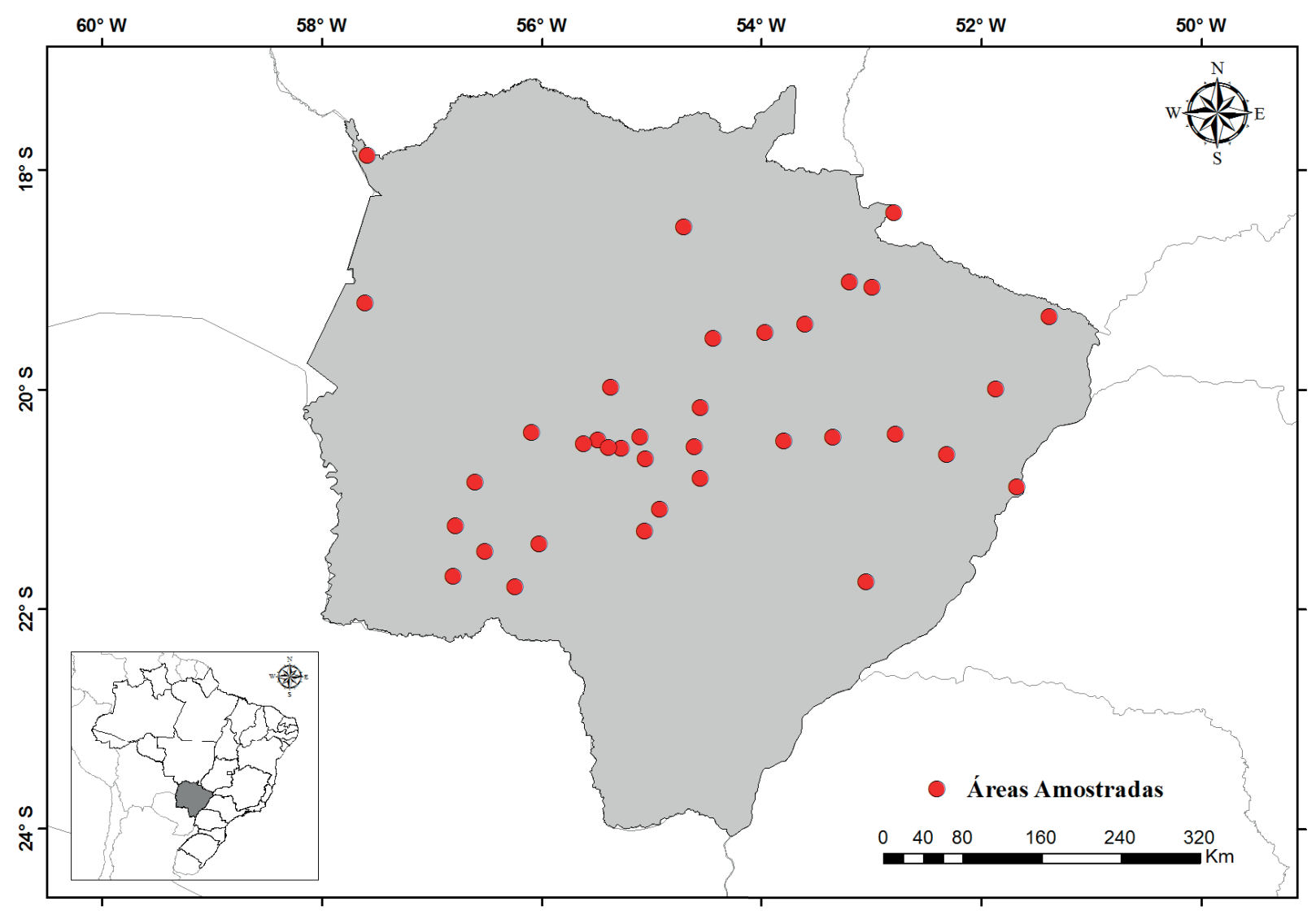

Fig. 1. Destaque em cinza para o estado de Mato Grosso do Sul e as áreas amostradas de Cerrado. 
dos autores no Cerrado do MS. O banco de dados do NeoTropTree (Oliveira-Filho 2014), consiste em um conjunto de banco de dados de listas florística de espécies arbóreas para mais de 2000 locais geo-referenciados compilados por meio de listagens originárias de publicações (periódicos, teses e dissertações) e registros de herbário (árvores aqui definido lenhosa plantas $>$ três metros de altura) e enriquecidas continuamente por novos registros encontrados em herbários e na literatura, particularmente nas monografias de revisão taxonômica. Todo protocolo sobre o NeoTropTree pode ser encontrado em http://prof. icb.ufmg.br/treeatlan/.

\section{RESULTADOS E DISCUSSÃO}

Foram registradas 546 espécies arbóreas distribuídas em 84 famílias botânicas e 255 gêneros (Quadro 1), dos quais três espécies [(Bauhinia leptantha Malme, Bougainvillea malmeana Heimerl e Cordia brasiliensis (I.M. Johnst.) Gottschling \& J.S.Mill)] segundo Oliveira-Filho (2014) e na lista de espécies da Flora do Brasil (2013), são exclusivas do Cerrado do MS. De acordo com o Livro Vermelho da Flora do Brasil - Plantas raras do Cerrado (Martinelli et al. 2014), Bauhinia leptantha foi classificada como ameaçada na categoria de vulnerável, e que tal fator estaria relacionado à perda do hábitat (Lima et al. 2014a).

As famílias destaque em riqueza de espécies foram Leguminosae com 87 espécies, Myrtaceae 43, Rubiaceae 31, Lauraceae 20, Annonaceae 19, Vochysiaceae e Malvaceae 17, Euphorbiaceae e Melastomataceae 15, Chrysobalanaceae e Sapindaceae com 14 e Arecaceae e Apocynaceae 13 espécies. A família Leguminosae reúne $24 \%$ das espécies listadas para a flora vascular do Cerrado (Mendonça et al. 2008), e as famílias destacadas no Cerrado do MS, tem sido representada como as mais diversas em vários trabalhos no bioma Cerrado (Gentry et al. 1997, Felfili et al. 2002, Ratter et al. 2003, Neri et al. 2007, Mendonça et al. 2008, Bueno et al. 2013a, b).

Os gêneros que se destacaram foram: Myrcia DC. (17 espécies), Eugenia L. (12 spp.), Aspidosperma Mart., Erythroxylum P. Browne e Ocotea Aubl. (10 spp.), Annona L. (nove spp), Byrsonima Rich. ex Kunth, Ficus L., Licania Aubl., Machaerium Pers. e Miconia Ruiz \& Pav. (oito espécies), Bauhinia L. e Vochysia Aubl. (sete spp.), Cordia L. e Qualea Aubl. (seis spp.), Casearia Jacq., Campomanesia Ruiz \& Pav., Coccoloba P. Browne, Handroanthus Mattos, Myrsine L. e Ouratea Aubl. com cinco espécies. Os gêneros Myrcia e Eugenia também são registrados em destaque para a Mata Atlântica (Oliveira-Filho \& Fontes 2000, Ivanauskas et al. 2001), e o exemplo do gênero Ficus tem importante representação nas florestas estacionais do estado de Mato Grosso do Sul (Damasceno-Junior et al. 2017). Outros gêneros como Aspidosperma, Casearia e Machaerium tem sido registrado com significativa ocorrência em Florestas Estacionais (Ratter et al. 1988, Damasceno-Junior et al. 2017) e Ocotea como uma das mais importantes da Amazônia, porém pouco representada nos cerrados (Gentry
1990). Já Byrsonima, Erythroxylum, Qualea e Vochysia, tem significativa importância no bioma Cerrado (Ratter et al. 2003, Bridgewater et al. 2004, Mendonça et al. 2008).

Dentre o total de espécies registradas, 98 (80\%) foram determinadas como espécies arbóreas dominantes no bioma Cerrado em um total de 121 espécies de acordo com Bridgewater et al. (2004), evidenciando que a flora arbórea do Cerrado do MS está bem representada em relação a flora arbórea do Cerrado no contexto geral do Bioma. Contudo, o compartilhamento da flora arbórea do Cerrado do MS em relação a flora de formações vegetacionais (biomas) adjacentes representam 37\% flora arbórea do Cerrado do MS (Fig. 2). Isso corrobora com Ratter et al. (2003) que relatam que as áreas marginais do Cerrado, como o caso do MS, ocorrem elementos florísticos de formações vegetais adjacentes, compondo a riqueza do Cerrado como um todo.

A característica do maior compartilhamento da flora do Cerrado com a Mata Atlântica, como registrado para o Cerrado do MS (18\% Mata Atlântica e 6\% Amazônia), também foi evidenciado por Oliveira-Filho \& Fontes (2000), ao analisarem padrões florísticos da flora arbórea na Mata Atlântica, na qual, o Cerrado compartilhou uma proporção muito maior (55\%) de sua flora com floresta atlântica do que a floresta amazônica (20\%). Já os dois grandes núcleos de Floresta Estacional Sazonalmente Seca da América do Sul, o Chaco e a Caatinga, apresentaram os menores compartilhamentos de espécies (Fig. 2). O Chaco, expressou um maior compartilhamento que a Caatinga ( $3,5 \%$ e $0,5 \%$ respectivamente), provavelmente em razão da proximidade geográfica entre as formações vegetacionais do Cerrado do MS, onde o oposto é registrado para a Caatinga. Oliveira-Filho et al. (2005), também relatam essa baixa proporção de espécies arbóreas compartilhadas entre Caatinga e Cerrado. Deve se levar em conta, que o trabalho atual está focado em fitofisionomias do Cerrado sentido amplo (Ribeiro \& Walter 2008) (Fig. 1), excluindo áreas de Florestas Estacional Decidual e Semidecidual por exemplo, na qual se consideradas outras fitofisionomias do Bioma Cerrado presentes no estado do MS, o número de espécies e o compartilhamento de espécies aumentaria significativamente com os biomas adjacentes.

Foram listadas 36 espécies características de solos férteis (Quadro 1). Na caracterização das províncias fitogeográficas do Cerrado, Ratter et al. (2003) descrevem uma forte tendência das províncias mais ocidental e a do centro oeste, serem dominadas por espécies características dos solos mesotróficos (mais férteis), por exemplo, Magonia pubescens A.St.-Hil., Astronium fraxinifolium Schott e Callisthene fasciculata (Spreng.) Mart., e a muito baixa ocorrência de espécies nos cerrados distróficos da província do Sul. A tendência de certas espécies no Cerrado estarem associadas com solos mesotróficos tem sido bem documentada (ver, por exemplo, Ratter et al. 1973, 1977, Furley \& Ratter 1988, Bueno et al. 2013b). Ainda, de acordo com Ratter (comunicação pessoal), grande parte dos cerradões levantados no estado do MS apresentam tipologia mesotrófica. O cerradão mesotrófico normalmente 
Quadro 1. Lista das famílias e espécies arbóreas registradas para o Cerrado do estado de Mato Grosso do Sul. * Espécies listadas como dominantes do Cerrado (Bridgewater et al. 2004); ** espécies características de solo férteis (Ratter et al. 1977, 2011, Furley \& Ratter 1988, Bueno et al. 2013b).

\begin{tabular}{|c|c|}
\hline Anacardiaceae & Arecaceae \\
\hline Astronium fraxinifolium Schott* & Acrocomia aculeata (Jacq.) Lodd. ex Mart.** \\
\hline Lithrea molleoides (Vell.) Engl. & A. glaucescens Lorenzi \\
\hline Myracrodruon urundeuva Allemão** & A. totai Mart. \\
\hline Spondias mombin L.** & Attalea phalerata Mart. ex Spreng. \\
\hline Tapirira guianensis Aubl.* & A. speciosa Mart. ex Spreng. \\
\hline Annonaceae & Bactris glaucescens Drude \\
\hline Annona amambayensis Hassl. ex R.E.Fr. & Butia paraguayensis (Barb.Rodr.) L.H.Bailey \\
\hline A. cacans Warm. & Euterpe edulis Mart. \\
\hline A. coriacea Mart.* & Mauritia flexuosa L.f. \\
\hline A. cornifolia A. St.-Hil. & Syagrus comosa (Mart.) Becc.* \\
\hline A. crassiflora Mart*. & S. flexuosa (Mart.) Becc.* \\
\hline A. emarginata (Schltdl.) H. Rainer & S. oleracea (Mart.) Becc. \\
\hline A. neosalicifolia H. Rainer & S. romanzoffiana (Cham.) Glassman \\
\hline A. nutans (R.E.Fr.) R.E.Fr. & Asteraceae \\
\hline A. sylvatica A.St.-Hil. & Eremanthus cinctus Baker \\
\hline Bocageopsis mattogrossensis (R.E.Fr.) R.E.Fr. & Gochnatia floribunda Cabrera \\
\hline Duguetia lanceolata A. St.-Hil. & G. polymorpha (Less.) Cabrera \\
\hline D. marcgraviana Mart. & G. pulchra Cabrera \\
\hline Guatteria australis A. St.-Hil. & Koanophyllon tinctorium Arruda ex H.Kost. \\
\hline G. rigida R.E.Fr. & Piptocarpha rotundifolia (Less.) Baker* \\
\hline G. sellowiana Schltdl. & Bignoniaceae \\
\hline Unonopsis guatterioides (A.DC.) R.E.Fr. & Handroanthus impetiginosus Mattos \\
\hline Xylopia aromatica (Lam.) Mart. & H. ochraceus (Cham.) Mattos* ** \\
\hline$X$. emarginata Mart. & H. selachidentatus (A.H.Gentry) S.O.Grose \\
\hline$X$. sericea A.St.-Hil. & H. serratifolius (Vahl) S.O.Grose \\
\hline Apocynaceae & H. umbellatus (Sond.) Mattos \\
\hline Aspidosperma cylindrocarpon Müll.Arg. & Jacaranda caroba (Vell.) A.DC. \\
\hline A. discolor A.DC. & J. cuspidifolia Mart. ex A.DC.** \\
\hline A. macrocarpon Mart. ${ }^{*} * *$ & J. puberula Cham. \\
\hline A. multiflorum A.DC. & Tabebuia aurea (Manso) Benth. \& Hook.f. ex *S.Moore \\
\hline A. nobile Müll.Arg. & T. insignis (Miq.) Sandwith \\
\hline A. pyrifolium Mart. & T. nodosa (Griseb.) Griseb. \\
\hline A. ramiflorum Müll.Arg. & T. roseoalba (Ridl.) Sandwith** \\
\hline A. subincanum Mart. ex A.DC.*** & Zeyheria montana Mart.* \\
\hline A. tomentosum Mart.* ** & Boraginaceae \\
\hline A. triternatum Rojas Acosta & Cordia alliodora (Ruiz \& Pav.) Oken \\
\hline Hancornia speciosa Gomes* & C. brasiliensis (I.M. Johnst.) Gottschling \& J.S. Mill. \\
\hline Himatanthus articulatus (Vahl) Woodson & C. glabrata (Mart.) A.DC. \\
\hline H. obovatus (Müll. Arg.) Woodson* & C. insignis Cham. \\
\hline Aquifoliaceae & C. sellowiana Cham. \\
\hline Ilex affinis Gardner & C. trichotoma (Vell.) Arrab. ex Steud. \\
\hline I. brasiliensis (Spreng.) Loes. & Burseraceae \\
\hline I. cerasifolia Reissek & Commiphora leptophloeos (Mart.) J.B. Gillet \\
\hline Araliaceae & Protium heptaphyllum (Aubl.) Marchand \\
\hline Aralia warmingiana (Marchal) J.Wen & P. grandifolium Engl. \\
\hline Schefflera calva (Cham.) Frodin \& Fiaschi & P. ovatum Engl. \\
\hline S. distractiflora (Harms) Frodin & Cactaceae \\
\hline S. malmei (Harms) Frodin & Brasiliopuntia brasiliensis (Willd.) A. Berger \\
\hline S. vinosa (Cham. \& Schltdl.) Frodin \& Fiaschi & Cereus bicolor Rizzini \& Mattos Filho \\
\hline Monvillea spegazzinii (F.A.C. Weber) Britton \& Rose & T. phaeocarpa Eichler \\
\hline Cannabaceae & Dilleniaceae \\
\hline Celtis iguanaea (Jacq.) Sarg. & Curatella americana L.* \\
\hline C. pubescens (Kunth) Spreng. & Davilla elliptica A.St.-Hil.* \\
\hline Trema micrantha (L.) Blume & D. grandiflora A.St.-Hil. \& Tul. \\
\hline Calophyllaceae & Ebenaceae \\
\hline Kielmeyera coriacea Mart. \& Zucc.* & Diospyros hispida A.DC.* \\
\hline
\end{tabular}


Quadro 1. Cont.

\begin{tabular}{|c|c|}
\hline K. rubriflora Cambess.* & D. poeppigiana A.DC. \\
\hline Capparaceae & D. sericea A.DC. \\
\hline Cynophalla mattogrossensis (Pilg.) Cornejo \& Iltis & Ericaceae \\
\hline Cardiopteridaceae & Gaylussacia brasiliensis (Spreng.) Meisn. \\
\hline Citronella gongonha (Mart.) R.A. Howard & Erythropalaceae \\
\hline Caricaceae & Heisteria ovata Benth. \\
\hline Jacaratia heptaphylla (Vell.) A.DC. & Erythroxylaceae \\
\hline Caryocaraceae & Erythroxylum anguifugum Mart. \\
\hline Caryocar brasiliense Cambess.* & E. citrifolium A.St.-Hil. \\
\hline Celastraceae & E. cuneifolium (Mart.) O.E. Schulz \\
\hline Maytenus aquifolia Mart. & E. daphnites Mart. \\
\hline M. floribunda Reissek & E. deciduum A.St.-Hil. \\
\hline Peritassa dulcis (Benth.) Miers & E. engleri O.E.Schulz \\
\hline Plenckia populnea Reissek* & E. patentissimum O.E. Schulz \\
\hline Salacia crassifolia (Mart. ex Schult.) G. Don* & E. pelleterianum A.St.-Hil. \\
\hline S. elliptica (Mart. ex Schult.) G. Don & E. suberosum A.St.-Hil.* \\
\hline Chrysobalanaceae & E. tortuosum Mart.* \\
\hline Couepia grandiflora (Mart. \& Zucc.) Benth. ex Hook.f.* & Euphorbiaceae \\
\hline Couepia uiti (Mart. \& Zucc.) Benth. ex Hook. f. & Actinostemon klotzschii (Didr.) Pax \\
\hline Hirtella glandulosa Spreng. & Alchornea castaneifolia (Humb. \& Bonpl. ex Willd.) A. Juss. \\
\hline H. gracilipes (Hook.f.) Prance & A. discolor Poepp. \& Endl. \\
\hline H. hoehnei Pilg. & Cnidoscolus paucistamineus (Pax) Pax \\
\hline Licania apetala (E.Mey.) Fritsch & Croton floribundus Spreng. \\
\hline L. blackii Prance & C. piptocalyx Müll. Arg. \\
\hline L. gardneri (Hook.f.) Fritsch & Gymnanthes boticario Esser, M.F.A. Lucena \& M.Alves \\
\hline L. humilis Cham. \& Schltdl.* & G. discolor (Spreng.) Müll. Arg. \\
\hline L. maguirei Prance & Mabea fistulifera Mart. \\
\hline L. minutiflora (Sagot) Fritsch & M. paniculata Spruce ex Benth. \\
\hline L. parvifolia Huber & Manihot caerulescens Pohl \\
\hline L. sclerophylla (Hook.f.) Fritsch & Maprounea brasiliensis A.St.-Hil. \\
\hline Parinari obtusifolia Hook.f. & Micrandra elata Müll.Arg. \\
\hline Clusiaceae & Pleradenophora membranifolia (Müll. Arg.) Esser \&. A.L. Melo \\
\hline Garcinia gardneriana (Planch. \& Triana) Zappi & Sapium glandulosum (L.) Morong \\
\hline Combretaceae & Icacinaceae \\
\hline Buchenavia tomentosa Eichler & Emmotum nitens (Benth.) Miers* \\
\hline Combretum duarteanum Cambess. & Lacistemataceae \\
\hline C. lanceolatum Pohl ex Eichler & Lacistema aggregatum (P.J.Bergius) Rusby \\
\hline C. leprosum Mart.** & L. hasslerianum Chodat \\
\hline C. mellifluum Eichler & Connaraceae \\
\hline Terminalia argentea (Cambess.) Mart.* & Connarus suberosus Planch.* \\
\hline T. fagifolia Mart.* & Rourea induta Planch.* \\
\hline T. glabrescens Mart. & Cunoniaceae \\
\hline Lamanonia ternata Vell. & B. rufa (Bong.) Steud.* \\
\hline Lamiaceae & Bowdichia virgilioides Kunth* \\
\hline Aegiphila brachiata Vell. & Calliandra foliolosa Benth. \\
\hline A. integrifolia (Jacq.) B.D. Jackson & C. harrisii (Lindl.) Benth. \\
\hline A. verticillata Vell. & Cenostigma macrophyllum Tul. \\
\hline A. vitelliniflora Walp. & Chamaecrista multiseta (Benth.) H.S.Irwin \& Barneby \\
\hline Hyptidendron canum (Pohl) Harley & C. orbiculata (*Benth.) H.S.Irwin \& Barneby \\
\hline Vitex cymosa Bert. ex Spreng.** & Copaifera langsdorffii Desf. \\
\hline$V$. polygama Cham. & C. malmei Harms \\
\hline Lauraceae & C. oblongifolia Mart. ex Hayne \\
\hline Aiouea piauhyensis (Meisn.) Mez & Dalbergia glaucescens (Mart. ex Benth) Benth \\
\hline A. trinervis Meisn. & D. miscolobium Benth. \\
\hline Aniba heringeri Vattimo-Gil & D. villosa (Benth.) Benth.* \\
\hline Cinnamomum haussknechtii (Mez) Kosterm. & Dimorphandra mollis Benth.** \\
\hline Cryptocarya moschata Nees \& Mart. ex Nees & Diptychandra aurantiaca Tul.* \\
\hline Mezilaurus crassiramea (Meisn.) Taub. ex Mez & Dipteryx alata Vogel $* * *$ \\
\hline
\end{tabular}


Quadro 1. Cont.

\begin{tabular}{|c|c|}
\hline M. vanderwerffii F.M. Alves \& J.B.Baitello & Erythrina similis Krukoff \\
\hline Nectandra amazonum Nees & E. verna Vell. \\
\hline N. gardneri Meisn. & Hymenaea martiana Hayne* \\
\hline N. lanceolata Nees & H. stigonocarpa Mart. ex Hayne** \\
\hline Ocotea corymbosa (Meisn.) Mez & Inga alba (Sw.) Willd. \\
\hline O. diospyrifolia (Meisn.) Mez & I. brevipes Benth. \\
\hline O. lancifolia $(\mathrm{Schott}) \mathrm{Mez}$ & I. disticha Benth.* \\
\hline O. minarum (Nees \& Mart.) Mez & Leptolobium dasycarpum Vogel \\
\hline O. pomaderroides (Meisn.) Mez & L. elegans Vogel \\
\hline O. pulchella (Nees \& Mart.) Mez & Lonchocarpus muehlbergianus Hassl. \\
\hline O. spectabilis (Meisn.) Mez & Luetzelburgia auriculata (Allemão) Ducke* \\
\hline O. spixiana $(\mathrm{Nees}) \mathrm{Mez}$ & Machaerium acutifolium Vogel \\
\hline O. velloziana (Meisn.) Mez & M. brasiliense Vogel \\
\hline O. velutina (Nees) Rohwer & M. eriocarpum Benth. \\
\hline Lecythidaceae & M. hirtum (Vell.) Stellfeld** \\
\hline Eschweilera nana (O.Berg) Miers & M. isadelphum (E.Mey) Amsh.* \\
\hline Lecythis lanceolata Poir. & M. opacum Vogel \\
\hline Leguminosae & M. paraguariense Hassl. \\
\hline Acosmium cardenasii H.S.Irwin \& Arroyo & M. saraense Rudd \\
\hline Albizia niopoides (Spruce ex Benth.) Burkart* & Macrolobium acaciifolium (Benth.) Benth. \\
\hline A. polycephala (Benth.) Killip ex Record & M. gracile Spruce ex Benth. \\
\hline Anadenanthera colubrina (Vell.) Brenan* ** & M. claussenii Benth. \\
\hline A. peregrina (L.) Speg.** & M. gemmulata Barneby \\
\hline Andira cujabensis Benth. *** & M. laticifera Rizzini \& A. Mattos \\
\hline A. fraxinifolia Benth. & M. pteridifolia Benth. \\
\hline A. inermis (W. Wright) DC. subsp. inermis & Muellera sericea (Micheli) M.J. Silva \& A.M.G. Azevedo \\
\hline A. vermifuga (Mart.) Benth.* & Parapiptadenia rigida (Benth.) Brenan \\
\hline Apuleia leiocarpa (Vogel) J. F. Macbr. & Peltogyne confertiflora (Mart. ex Hayne) Benth.* \\
\hline Ateleia guaraya Herzog & Plathymenia reticulata Benth.** \\
\hline Bauhinia brevipes Vogel & Platymiscium floribundum Vogel* \\
\hline B. holophylla (Bong.) Steud. & Platypodium elegans Vogel \\
\hline B. leptantha Malme & Poecilanthe parviflora Benth. \\
\hline B. mollis (Bong.) D.Dietr. & Prosopis rubriflora Hassl. \\
\hline P. ruscifolia Griseb. & E. pubescens (Mart. \& Zucc.) Schott \& Endl.*** \\
\hline Pterodon emarginatus Vogel* & Guazuma ulmifolia Lam. \\
\hline P. pubescens (Benth.) Benth.** & Helicteres brevispira A.St.-Hil.* ** \\
\hline Pterogyne nitens Tul. & H. corylifolia Nees \& Mart.** \\
\hline Riedeliella graciliflora Harms & Luehea divaricata Mart. \\
\hline Senegalia polyphylla (DC.) Britton \& Rose & L. microcarpa R.E.Fr. \\
\hline Senna rugosa (G.Don) H.S.Irwin \& Barneby & L. paniculata Mart. \& Zucc.*** \\
\hline S. silvestris (Vell.) H.S.Irwin \& Barneby & Pseudobombax grandiflorum (Cav.) A. Robyns \\
\hline S. velutina (Vogel) H.S.Irwin \& Barneby & P. heteromorphum (Kuntze) A.Robyns \\
\hline S. adstringens (Mart.) Coville & P. marginatum (A.St.-Hil.) A.Robyns \\
\hline S. polyphyllum Mart. & P. tomentosum (Mart. \& Zucc.) A. Robyns*** \\
\hline S. rotundifolium Mart. & Sterculia striata A.St.-Hill. \& Naudin** \\
\hline Swartzia apetala Raddi* & Marcgraviaceae \\
\hline Tachigali aurea Tul.** & Norantea guianensis (Aubl.) Choisy \\
\hline T. rubiginosa (Mart. ex Tul.) Oliveira-Filho & Melastomataceae \\
\hline T. subvelutina (Benth.) Oliveira-Filho & Graffenrieda weddellii Naudin \\
\hline T. vulgaris L.G. Silva \& H.C. Lima* ** & Miconia albicans (Sw.) Triana \\
\hline Vatairea macrocarpa (Benth.) Ducke** & M. elegans Cogn. \\
\hline Zygia cataractae (Kunth) L.Rico & M. ferruginata DC.* \\
\hline Z. pithecolobioides (O. Kuntze) Barneby \& J. W. Grimes & M. latecrenata (DC.) Naudin \\
\hline Loganiaceae & M. leucocarpa DC. \\
\hline Antonia ovata Pohl & M. ligustroides (DC.) Naudin \\
\hline Strychnos brasiliensis (Spreng.) Mart. & M. paucidens DC \\
\hline S. pseudoquina A.St.-Hil.* & M. stenostachya DC. \\
\hline Lythraceae & Mouriri elliptica Mart.* \\
\hline
\end{tabular}


Quadro 1. Cont.

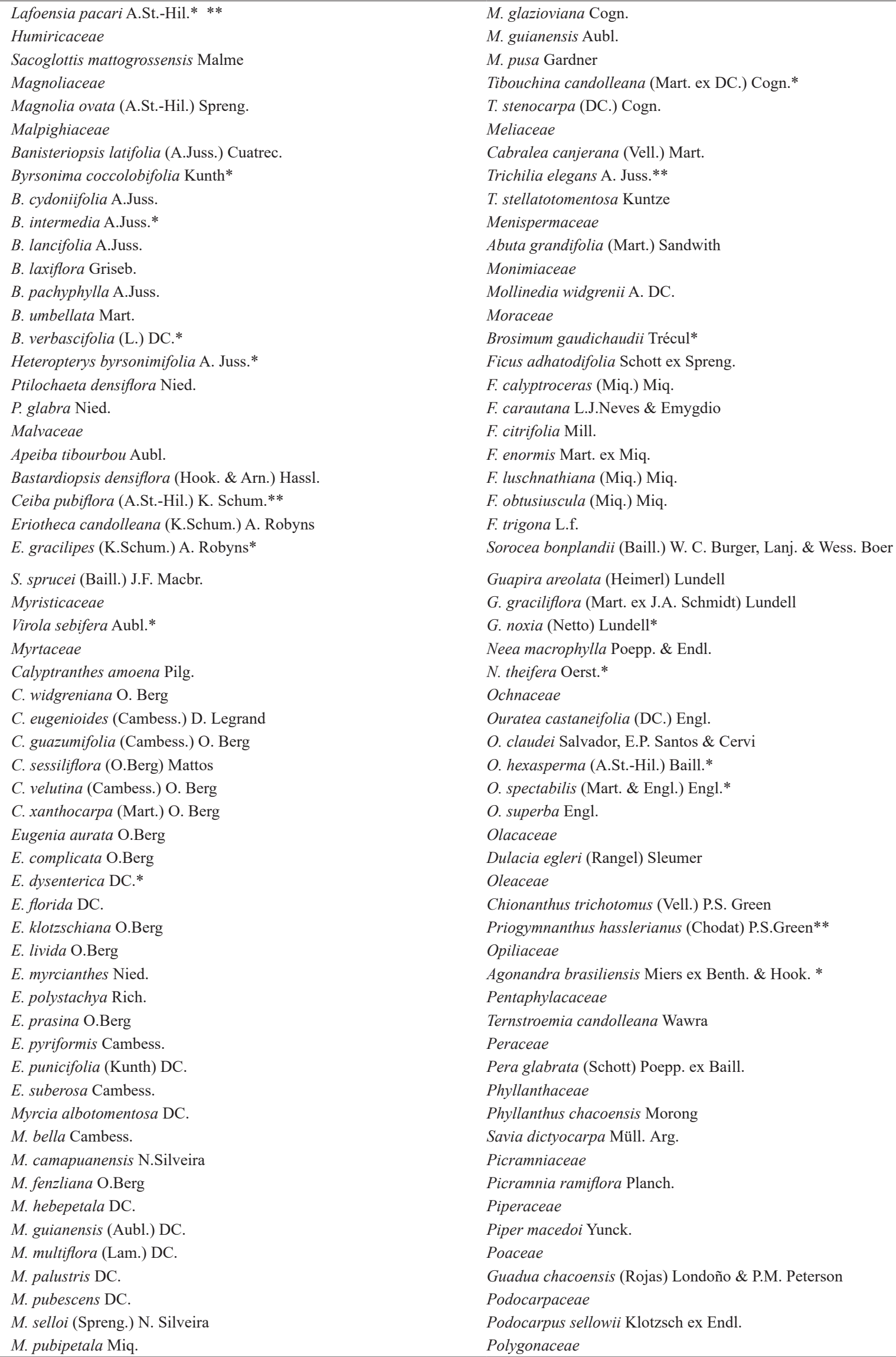

M. glazioviana Cogn.

M. guianensis Aubl.

M. pusa Gardner

Tibouchina candolleana (Mart. ex DC.) Cogn.*

T. stenocarpa (DC.) Cogn.

Meliaceae

Cabralea canjerana (Vell.) Mart.

Trichilia elegans A. Juss.**

T. stellatotomentosa Kuntze

Menispermaceae

Abuta grandifolia (Mart.) Sandwith

Monimiaceae

Mollinedia widgrenii A. DC.

Moraceae

Brosimum gaudichaudii Trécul*

Ficus adhatodifolia Schott ex Spreng.

F. calyptroceras (Miq.) Miq.

F. carautana L.J.Neves \& Emygdio

F. citrifolia Mill.

F. enormis Mart. ex Miq.

F. luschnathiana (Miq.) Miq.

F. obtusiuscula (Miq.) Miq.

F. trigona L.f.

Sorocea bonplandii (Baill.) W. C. Burger, Lanj. \& Wess. Boer

Guapira areolata (Heimerl) Lundell

G. graciliflora (Mart. ex J.A. Schmidt) Lundell

G. noxia (Netto) Lundell*

Neea macrophylla Poepp. \& Endl.

$N$. theifera Oerst.*

Ochnaceae

Ouratea castaneifolia (DC.) Engl.

O. claudei Salvador, E.P. Santos \& Cervi

O. hexasperma (A.St.-Hil.) Baill.*

O. spectabilis (Mart. \& Engl.) Engl.*

O. superba Engl.

Olacaceae

Dulacia egleri (Rangel) Sleumer

Oleaceae

Chionanthus trichotomus (Vell.) P.S. Green

Priogymnanthus hasslerianus (Chodat) P.S.Green**

Opiliaceae

Agonandra brasiliensis Miers ex Benth. \& Hook. *

Pentaphylacaceae

Ternstroemia candolleana Wawra

Peraceae

Pera glabrata (Schott) Poepp. ex Baill.

Phyllanthaceae

Phyllanthus chacoensis Morong

Savia dictyocarpa Müll. Arg.

Picramniaceae

Picramnia ramiflora Planch.

Piperaceae

Piper macedoi Yunck.

Poaceae

Guadua chacoensis (Rojas) Londoño \& P.M. Peterson

Podocarpaceae

Podocarpus sellowii Klotzsch ex Endl.

Polygonaceae 
Quadro 1. Cont.

\begin{tabular}{|c|c|}
\hline M. splendens (Sw.) DC. & Coccoloba cujabensis Wedd. \\
\hline M. tomentosa (Aubl.) DC. & C. guaranitica Hassler \\
\hline M. uberavensis Berg & C. marginata Benth. \\
\hline M. variabilis DC. & C. mollis Casar. \\
\hline M. venulosa DC. & C. obtusifolia Jacq. \\
\hline M. vestita DC. & Ruprechtia brachysepala Meisn. \\
\hline Myrciaria floribunda (H.West ex Willd.) O. Berg & R. exploratricis Sandwith \\
\hline Psidium acutangulum DC. & R. triflora Griseb. \\
\hline P. guineense Sw. & Triplaris gardneriana Weddell \\
\hline P. myrsinites DC. & Primulaceae \\
\hline P. myrtoides O.Berg & Myrsine coriacea (Sw.) R.Br. ex Roem. \& Schult. \\
\hline P. striatulum DC. & M. leuconeura Mart. \\
\hline Siphoneugena densiflora O.Berg & M. guianensis (Aubl.) Kuntze* \\
\hline Nyctaginaceae & M. matensis (Mez) Otegui \\
\hline Bougainvillea malmeana Heimerl & M. umbellata Mart. \\
\hline B. praecox Griseb & Proteaceae \\
\hline Euplassa inaequalis (Pohl) Engl. & Z. rigidum Humb. \& Bonpl. ex Willd.** \\
\hline Roupala montana Aubl.* & Salicaceae \\
\hline Rhamnaceae & Casearia decandra Jacq. \\
\hline Rhamnidium elaeocarpum Reissek $* * *$ & C. gossypiosperma Briq. \\
\hline Rosaceae & C. grandiflora Cambess.** \\
\hline Prunus myrtifolia (L.) Urb. & C. rupestris Eichler \\
\hline Rubiaceae & C. sylvestris Sw.* ** \\
\hline Alibertia edulis (Rich.) A.Rich. ex DC.* & Sapindaceae \\
\hline A. oligantha K.Schum. & Allophylus edulis (A.St.-Hil., Cambess. \& A. Juss.) Hieron. ex Niederl. \\
\hline Calycophyllum multiflorum Griseb. & Allophylus pauciflorus Radlk. \\
\hline Chomelia obtusa Cham. \& Schltdl. & A. sericeus (Cambess.) Radlk. \\
\hline C. pohliana Müll. Arg. & Averrhoidium paraguaiense Radlk. \\
\hline Cordiera macrophylla Kuntze & Cupania castaneifolia Mart. \\
\hline C. sessilis (Vell.) Kuntze** & C. oblongifolia Mart. \\
\hline Coussarea hydrangeifolia (Benth.) Müll. Arg. & C. tenuivalvis Radlk. \\
\hline C. platyphylla Müll.Arg. & C. vernalis Cambess. \\
\hline Faramea latifolia (Cham. \& Schltdl.) DC. & Dilodendron bipinnatum Radlk.* ** \\
\hline F. sessilifolia (Kunth) DC. & Diplokeleba floribunda N.E. Brown \\
\hline Ferdinandusa elliptica Pohl & Magonia pubescens A.St.-Hil.* ** \\
\hline F. rudgeoides (Benth.) Wedd. & Matayba guianensis Aubl. \\
\hline F. speciosa Pohl & Melicoccus lepidopetalus Radlk. \\
\hline Genipa americana $\mathrm{L}$. & Talisia esculenta (A.St.-Hil.) Radlk.** \\
\hline Guettarda pohliana Müll.Arg. & Sapotaceae \\
\hline G. viburnoides Cham. \& Schltdl.* ** & Chrysophyllum marginatum (Hook. \& Arn.) Radlk. \\
\hline Ladenbergia cujabensis Klotzsch & Micropholis venulosa (Mart. \& Eichler) Pierre \\
\hline L. graciliflora K. Schum. & Pouteria gardneri (Mart. \& Miq.) Baehni** \\
\hline Machaonia brasiliensis (Hoff. ex H.) Cham. \& Schltdl. & P. gardneriana (A.DC.) Radlk. \\
\hline Palicourea rigida Kunth* & P. ramiflora (Mart.) Radlk.*** \\
\hline Psychotria anceps Kunth & P. torta (Mart.) Radlk.* \\
\hline Randia armata (Sw.) DC. & Simaroubaceae \\
\hline R. calycina Cham. & Simaba maiana Casar. \\
\hline Remijia ferruginea DC. & Simarouba versicolor A.St.-Hil.* \\
\hline Rudgea jasminoides (Cham.) Müll. Arg. & Siparunaceae \\
\hline Simira corumbensis (Stand1.) Steyerm. & Siparuna brasiliensis (Spreng.) A.DC. \\
\hline S. hexandra (S.Moore) Steyerm. & S. guianensis Aubl.* \\
\hline Sphinctanthus microphyllus K.Schum. & Solanaceae \\
\hline Tocoyena formosa (Cham. \& Schltdl.) K. Schum.* & Cestrum axillare Vell. \\
\hline Rutaceae & Solanum crinitum Lam. \\
\hline Balfourodendron riedelianum (Engl.) Engl. & S. gomphodes Dunal \\
\hline Esenbeckia febrifuga (A.St.-Hil.) A.Juss. ex Mart. & S. lycocarpum A.St.-Hil. \\
\hline E. leiocarpa Engl. & Styracaceae \\
\hline Helietta apiculata Benth. & S. camporum Pohl \\
\hline
\end{tabular}


Quadro 1. Cont.

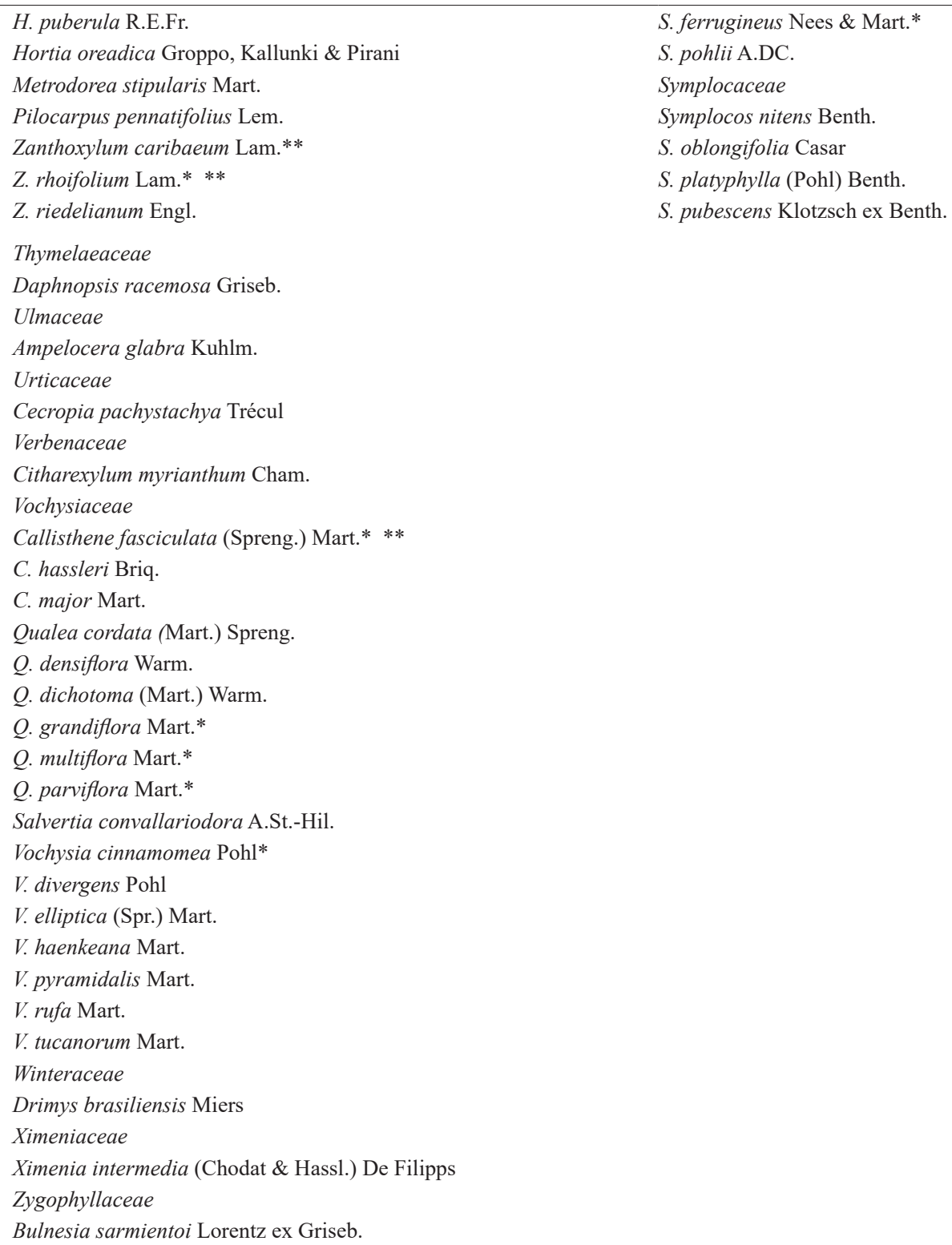

S. ferrugineus Nees \& Mart.*

S. pohlii A.DC.

Symplocaceae

Symplocos nitens Benth.

S. oblongifolia Casar

S. platyphylla (Pohl) Benth.

S. pubescens Klotzsch ex Benth.

ocorre como uma faixa de transição em solos intermediários de fertilidade, isto é, entre cerradão distrófico ou cerrado stricto sensu com a Floresta Estacional Decidual (Mata Seca) (Ratter et al. 1977, Furley \& Ratter 1988, Bueno et al. 2013b). Em Porto Murtinho esse tipo de formação ocorre em morros com afloramento rochoso, lado a lado com as florestas estacionais deciduais e as formações chaquenhas.

Quando em comparação da riqueza de espécies arbóreas do Cerrado do MS com outros estados provenientes da distribuição do bioma Cerrado, o estado do MS apresenta uma menor riqueza (Ratter et al. 1997, Costa \& Araújo 2001, Felfili et al. 2002, Bridgewater et al. 2004). Ratter et al. (1997) argumentam que áreas de cerrado na região do Alto Araguaia no estado do Mato Grosso, Tocantins e Distrito Federal, ou seja, a região core é que apresentam as maiores riquezas de espécies em relação às áreas marginais e disjuntas do bioma Cerrado. Esse fato pode estar relacionado com a questão das flutuações climáticas do Quaternário, em que a região do MS é representada com baixa adequabilidade (menor estabilidade) em relação a região central que apresentou clima mais estável durante o período do último glacial máximo (Bueno et al. 2016). Tal evento distingue a região do MS como de recente distribuição das espécies do Cerrado, em relação aos estados de maior riqueza de espécies, que se localizaram nas áreas mais estáveis (região central do Brasil), frente as flutuações climáticas do Quaternário. Este evento é corroborado quando levado em conta estudos filogeográficos de espécies arbóreas comuns do Cerrado como, Caryocar brasiliense Cambess., Hymenaea stigonocarpa Mart. ex Hayne, Plathymenia reticulata Benth., Tabebuia aurea (Manso) Benth. \& Hook.f. ex S.Moore e Mauritia flexuosa 


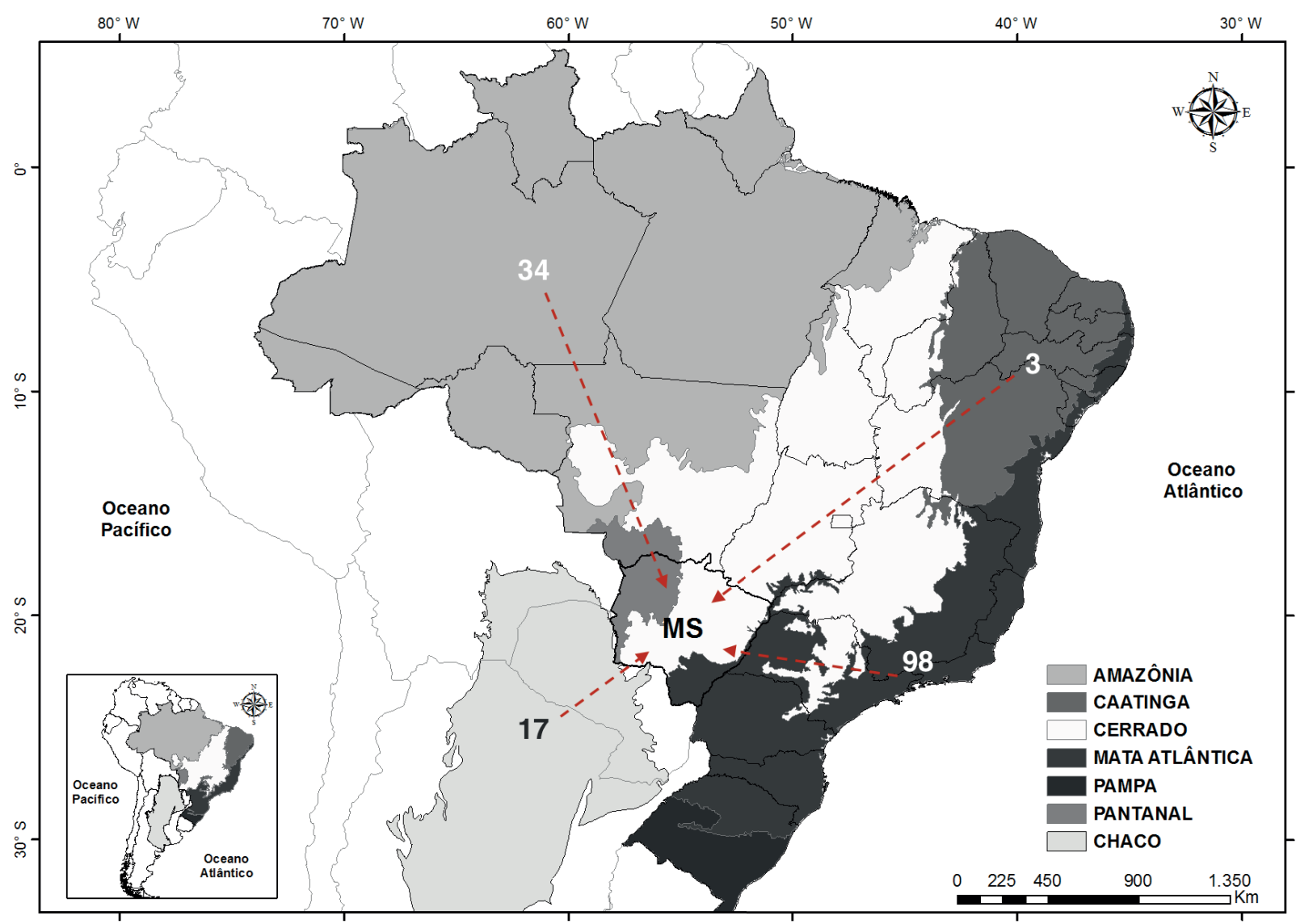

Fig. 2. Contribuição da flora arbórea das formações adjacentes (Biomas brasileiros e Chaco) indicado pelas setas e números para a composição da flora arbórea do Cerrado de Mato Grosso do Sul (MS).

L.f., que demonstram a região central do Brasil, ou a região core do Cerrado como a de maior diversidade genética, indicando esta região como a de maior estabilidade durante as flutuações climáticas do Quaternário (Collevati et al. 2003, Ramos et al. 2007, Novaes et al. 2010, Rosa 2012, Lima et al. 2014b).

Deste modo, concluímos que a flora arbórea do Cerrado do MS apresenta uma riqueza considerável de espécies arbóreas, mesmo considerando os baixos índices de coleta no estado. Um exemplo disso, temos áreas de características peculiares como o cerrado rupícola, que apresentou apenas uma área amostrada, na qual essa formação é bem representativa em algumas áreas como, na Serra de Maracajú (que se estende cruzando o estado na direção sudoeste - nordeste), Serra das Araras (região de Alcinópolis e Costa Rica), Serra Preta (Sonora) e regiões como Serra do Amolar, na planície pantaneira. Dessa forma, é urgente a realização de mais estudos e de incentivos a coletas em todas as fitofisionomias do Cerrado no estado, devido a forte pressão antrópica que este bioma está sendo imposto. Seguindo esse foco, o projeto como o BiotaMS, tem um papel fundamental, pois está proporcionando melhora no conhecimento sobre a distribuição e abundância das espécies arbóreas no Cerrado do estado de Mato Grosso do Sul. Assim, a principal tarefa em relação à flora do estado e particularmente a flora arbórea do Cerrado, é a de ampliar o conhecimento da biodiversidade, aumentar os números de coletas e focar em regiões ainda não coletadas ou com baixo número de coletas.

\section{AGRADECIMENTOS}

Aos órgãos financiadores que contribuíram com financiamento do projeto NeoTropTree (TreeAtlan), e ao Conselho Nacional de Desenvolvimento Científico e Tecnológico, pela bolsa de Pós-doutorado (PDJ 151002/2014-2) de Marcelo Leandro Bueno e a bolsa produtividade para Geraldo A. Damasceno-Junior.

\section{REFERÊNCIAS}

Ab'Saber, A.N. 2003. Os Domínios de Natureza no Brasil: potencialidades paisagísticas. Ateliê Editorial, São Paulo. 159 p.

Adámoli, J., Macêdo, J., Azevedo, L.G. \& Netto, J.M. 1987. Caracterização da região dos Cerrados. In Solos dos Cerrados: tecnologias e estratégias de manejo (W.J. Goedert, ed.). Nobel, São Paulo, p. 33-98.

Alves, F.M., Lehn, C.R., Damasceno-Júnior, G.A., Sartori, A.L.B.; Pott, A., Pott, V.J., Bortolotto, I.M., Ishii, I.H., Salis, S.M.; Urbanetz, C., Bueno, M.L. \& Sciamarelli, A. 2017. Coleções botânicas do estado de Mato Grosso do Sul: situação atual e perspectivas. Iheringia, Série Botânica 73(supl.):93-100. 
Bridgewater, S., Ratter, J.A. \& Ribeiro, J.F. 2004. Biogeographic patterns, b-diversity and dominance in the cerrado biome of Brazil. Biodiversity and Conservation 13:2295-2318.

Bueno, M. L.; Pennington, R. T; Dexter, K. G.; Kamino, L. H. Y.; Pontara, V.; Neves, D. M.; Ratter, J. A. \& Oliveira-Filho, A. T. 2016. Effects of Quaternary climatic fluctuations on the distribution of Neotropical savanna tree species. Ecography 40: 403-414.

Bueno, M.L., Neves, D.R.M., Souza, A.F., Junior, E.O., Damasceno-Junior, G.A., Pontara, V., Laura, V. A. \& Ratter, J. A. 2013a. Influence of edaphic factors on the floristic composition of an area of cerradão in the Brazilian central-west. Acta Botanica Brasilica 27(2): 445-455.

Bueno, M.L., Neves, D.R.M., Oliveira-Filho, A.T., Lehn, C.R. \& Ratter, J.A. 2013b. A Study In an Area of Transition Between Seasonally Dry Tropical Forest And Mesotrophic Cerradão, In Mato Grosso do Sul, Southwestern Brazil. Edinburgh Journal of Botany 70(3): 469-486.

Collevati, R.G.; Grattapaglia, D. \& Hay J.D. 2003. Evidences for multiple maternal lineages of Caryocar brasiliense populations in the Brazilian Cerrado based on the analysis of chloroplast DNA sequences and microsatellite haplotype variation. Molecular Ecology 12: 105-115.

Costa, A.A. \& Araújo, G.M. 2001. Comparação da vegetação arbórea de cerradão e cerrado na Reserva do Panga, Uberlândia, Minas Gerais. Acta Botanica Brasilica 15(1): 63-72.

Damasceno-Junior, G.A., Pott, A., Neves, D.R.M., Sciamarelli, A. \& Fina, B.G. 2015. Flora lenhosa de Florestas estacionais do estado de Mato Grosso do Sul: estado da arte. Iheringia, Série Botânica 73(supl.):65-79.

Felfili, J.M., Nogueira, P.E., Júnior, M.C.S., Marimon, B.S. \& Delitti, W.B.C. 2002. Composição florística e fitossociologia de um cerrado sentido restrito no município de Água Boa-MT. Acta botanica Brasilica 16(1): 103-112.

Fundo Mundial para a Natureza. 1995. De grão em grão o Cerrado perde espaço. Impactos do Processo de Ocupação. World Wide Fund for Nature/Fundação Pró-Cerrado. Brasília. 66 p.

Furley P.A. \& Ratter, J.A. 1988. Soil resources and plant communities of the central Brazililian cerrado and their development. Journal of Biogeography 15: 97-108.

Gentry, A.H. 1990. Floristic similarities and differences between Southern Central America and Upper and Central Amazonia. In Four neotropical rainforests. (A.H. Gentry ed.). Yale University Press, London, p. 141-157.

Gentry, A.H., Herrera-MacBride, O., Huber, O., Nelson, B.W. \& Villamil, C.B. 1997. Regional overview: South America. In Centres of plant diversity (V.H. Heywood \& S.D, Davis, cords.). World Wide Fund for Nature/ International Union for Conservation of Nature, Cambridge, p. 269-307

Haridasan, M. 2000. Nutrição mineral de plantas nativas do cerrado. Revista Brasileira de Fisiologia Vegetal 12: 54-64.

Instituto Brasileiro de Geografia e Estatística-IBGE 2004. Mapa de biomas brasileiros. Rio de Janeiro: IBGE, Escala 1:5.000.000.

Ivanauskas, N.M., Monteiro, R. \& Rodrigues, R.R. 2001. Levantamento florístico de trecho de floresta Atlântica em Pariquera-Açu, São Paulo, Brasil. Naturalia 26: 97-129.

Lima, H., Souza, E.R., Rando, J.G., Bortoluzzi, R., Flores, A.S., Limas, L.C.P., Dutra, V., Vivas, C., Perez, A.P.F., Araújo, L., Mouton, L., Amaro, R. \& Serrano, T. 2014a. Fabaceae. In Livro Vermelho da flora do Brasil - Plantas raras do Cerrado (G. Martinelli, T. Messina \& L. S. Filho, eds.). Instituto de Pesquisas Jardim Botânico do Rio de Janeiro, Centro Nacional de conservação da flora, p. 123-140.

Lima, N.E, Lima-Ribeiro, M.S., Tinoco, C.F., Terribile, L.C. \& Collevatti, R.G. 2014b. Phylogeography and ecological niche modelling, coupled with the fossil pollen record, unravel the demographic history of a Neotropical swamp palm through the Quaternary. Journal of Biogeography 41: 673-686.

Lista de Espécies da Flora do Brasil 2013. Jardim Botânico do Rio de Janeiro. http://floradobrasil.jbrj.gov.br/. Acessado em 15.03.2015.

Lopes, A.S. 1984. Solos sob cerrado: características, propriedades e manejo. Potafos, Piracicaba. 162p.

Martinelli, G. \& Martins, E. 2010. Panorama nacional sobre espécies ameaçadas de extinção da flora brasileira. In Diversidade Vegetal Brasileira: conhecimento, conservação e uso (M.L. Asby, F.D.A. Matos, \& I.L. Amaral, orgs.). Sociedade Botânica do Brasil, Manaus, p. $592-595$.
Martinelli, G., Messina, T. \& Filho, L.A.S. 2014. Livro vermelho da flora do Brasil - Plantas raras do Cerrado (G. Martinelli, T. Messina \& Luiz A. S. Filho, orgs). Instituto de Pesquisas Jardim Botanico do Rio de Janeiro, Centro Nacional de conservação da flora, Rio de Janeiro. 320 p.

Mendonça, R.C., Felfili, J.M., Walter, B.M.T., Silva-Jr., M.C., Rezende, A.V., Filgueiras, T.S., Nogueira, P.E. \& Fagg, C.W. 2008. Flora vascular do cerrado: Checklist com 12.356 espécies. In Cerrado: ecologia e flora (S.M. Sano, S.P. Almeida \& J.F. Ribeiro, eds.). Embrapa, Planaltina, p.417-1279.

Ministério do Meio Ambiente- MMA 2009. Relatório técnico de monitoramento do desmatamento no Bioma Cerrado, 2002 a 2008: Dados revisados. Brasília: Ministério do Meio Ambiente. 69 p.

Mittermeier, R.A., Myers, N. \& Mittermeier, C.G. 1999. Hotspots: earts biologicaly richest and most endangered terrestrial ecoregions. Mexico City. Conservation International and Agrupacion Sierra Madre, Monterrey. $430 \mathrm{p}$.

Myers, N., Mittermeier, R.A., Mittermeier, C.G., Fonseca, G.A.B. \& Kent, J. 2000. Biodiversity hotspots for conservation priorities. Nature, 403 (6772): 853-858.

Neri, A.V., Meira Neto, J.A.A., Silva, A.F. da, Martins, S.V. \& Batista, M.L. 2007. Análise da estrutura de uma comunidade lenhosa em área de Cerrado Sensu Stricto no município de Senador Modestino Gonçalves, Norte de Minas Gerais, Brasil. Revista Árvore 31(1): 123-134.

Novaes, R.M.L., Filho, J.P.L., Ribeiro, R.A. \& Lovato, M.B. 2010. Phylogeography of Plathymenia reticulata (Leguminosae) reveals patterns of recent range expansion towards northeastern Brazil and southern Cerrados in Eastern Tropical South America. Molecular Ecology 19: 985-998.

Oliveira-Filho, A.T. \& Fontes, M.A.L. 2000. Patterns of floristic differentiation among Atlantic Forests in Southeastern Brazil, and the influence of climate. Biotropica 32: 793-810.

Oliveira-Filho, A.T., Neto, E.T., Carvalho, W.A.C., Werneck, M., Brina, A.E., Vidal, C.V., Rezende, S.C. \& Pereira, J.A.A. 2005. Análise florística do compartimento arbóreo de áreas de floresta atlântica sensu lato na região das bacias do leste. Rodriguésia 56: 185-235.

Oliveira-Filho, A.T. 2009. Classificação das fitofisionomias da América do Sul cisandina tropical e subtropical: proposta de um novo sistema - prático e flexível - ou uma injeção a mais de caos? Rodriguésia 60 (2): 237-258.

Oliveira-Filho, A.T. 2014. NeoTropTree, Flora arbórea da Região Neotropical: Um banco de dados envolvendo biogeografia, diversidade e conservação. Universidade Federal de Minas Gerais. http://www. icb.ufmg.br/treeatlan/. Acessado em 12.01.15.

Peixoto, A.L. 2003. Coleções biológicas de apoio ao inventário, uso sustentável e conservação da biodiversidade. Rio de Janeiro: Instituto de Pesquisas Jardim Botânico do Rio de Janeiro.

Pott, A. \& Pott, V.J. 2003. Espécies de Fragmentos Florestais em Mato Grosso do Sul. In Fragmentação florestal e alternativas de desenvolvimento rural na região Centro-Oeste. (R.B. da Costa, ed.). Universidade Católica Dom Bosco, Campo Grande. p. 26-52.

Ramos, A.C.S., Lemos-Filho, J.P., Ribeiro, R.A., Santos, F.R. \& Lovato, M.B. 2007. Phylogeography of the tree Hymenaea stigonocarpa (Fabaceae: Caesalpinioideae) and the influence of Quaternary climate changes in the Brazilian Cerrado. Annals of Botany 100: 1219-1228.

Ratter, J.A., Richards, P.N., Argente, G.E. \& Giford, D.R.G. 1973. Observations on the vegetation of northeastern Mato Grosso. Philosophical Transaction for the Royal Society of London, Series B.; Biological Sciences 226 (880): 449-492.

Ratter, J.A., Askew, G.P., Montgomery, R.F. \& Gifford, D.R. 1977. Observações adicionais sobre o cerradão de solos mesotróficos no Brasil central. In IV Simpósio sobre o Cerrado: Bases para a Utilização Agropecuária. (M.G. Ferri, ed.). Editora da Universidade de São Paulo, São Paulo, p.303-316.

Ratter, J.A., Ribeiro, J.F. \& Bridgewater, S. 1997. The brazilian cerrado vegetation and threats to its biodiversity. Annals of Botany 80: 223-230.

Ratter, J.A., Pott, A., Pott, V.J., Cunha; C.N. \& Haridasan, M. 1988. Observations on woody vegetation types in the Pantanal and at Corumbá, Brazil. Notes Royal Botanic Garden Edinburgh 45: 503-525. 
Ratter, J.A., Bridgewater, S. \& Ribeiro, J.F. 2003. Analysis of the floristic composition of the Brazilian cerrado vegetation iii: comparison of the woody vegetation of 376 areas. Edinburgh Journal of Botany 60: 57-109.

Reatto A., Correia, J.R. \& Spera, S.T. 1998. Solo do bioma Cerrado: aspectos pedológicos. In Cerrado: ambiente e flora. (S.M. Sano, S.P. Almeida \& J.F. Ribeiro, eds.). Embrapa Cerrados, Planaltina, p.47-86.

Ribeiro, J.F. \& Walter, B.M.T. 2008. As principais fitofisionomias do Bioma Cerrado. In Cerrado: ecologia e flora (S.M. Sano, S.P. Almeida \& J.F. Ribeiro, eds.). Embrapa Cerrados, Planaltina. p.151 -212.

Rizzini, C.T. 1979. Tratado de fitogeografia do Brasil. v.2. Aspectos ecológicos. Hucitec/Edusp, São Paulo. 327p.

Rosa, F.F. 2012 Diversidade Genética e Conservação de Tabebuia Aurea (Silva Manso) Benth \& Hook. F. Ex S. Moore (Bignoniaceae), uma espécie arbórea do Cerrado. Dissertação 59f., Pontifícia Universidade Católica de Goiás, Brasil.

Scarano, F.R., Ceotto, P., Medeiros, R. \& Mittermeier, R.A. 2014. O bioma Cerrado: conservação e ameaças. In Livro Vermelho da flora do Brasil - Plantas raras do Cerrado (G. Martinelli, T. Messina \& L. S. Filho, eds.). Instituto de Pesquisas Jardim Botânico do Rio de Janeiro, Centro Nacional de conservação da flora , p. 21-24.

Shepherd, G.J. 2003. Plantas terrestres: Versão preliminar. Relatório de Avaliação do estado do conhecimento da diversidade biológica do Brasil. $60 \mathrm{p}$.

Silva, J.M.C. \& Bates, J.M. 2002. Biogeographic patterns and conservation in the South american cerrado: a tropical savanna hotspot. bioScience 52: $225-233$ 